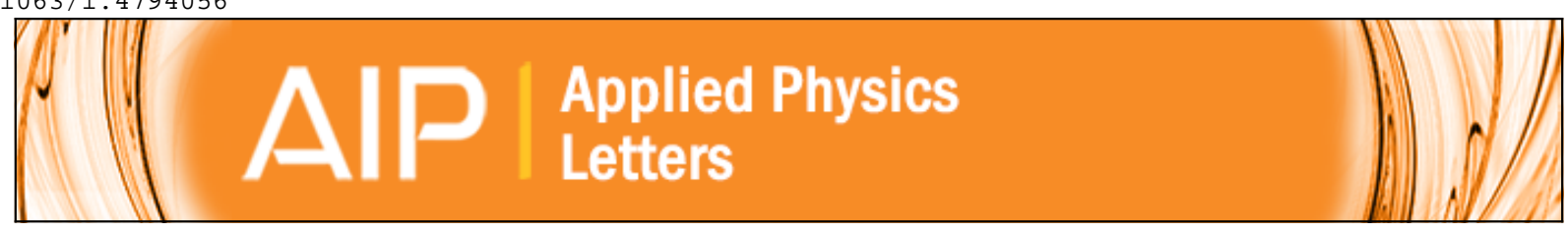

Giant magnetoelectric effect in self-biased laminates under zero magnetic field

Menghui Li, Zhiguang Wang, Yaojin Wang, Jiefang Li, and D. Viehland

Citation: Applied Physics Letters 102, 082404 (2013); doi: 10.1063/1.4794056

View online: http://dx.doi.org/10.1063/1.4794056

View Table of Contents: http://scitation.aip.org/content/aip/journal/apl/102/8?ver=pdfcov

Published by the AIP Publishing

AlP Re-register for Table of Content Alerts 


\title{
Giant magnetoelectric effect in self-biased laminates under zero magnetic field
}

\author{
Menghui Li, ${ }^{a)}$ Zhiguang Wang, Yaojin Wang, Jiefang Li, and D. Viehland \\ Department of Materials Science and Engineering, Virginia Tech, Blacksburg, Virginia 24061, USA
}

(Received 8 January 2013; accepted 18 February 2013; published online 27 February 2013)

\begin{abstract}
A giant magnetoelectric (ME) effect in self-biased annealed Metglas $/ \mathrm{Pb}(\mathrm{Zr}, \mathrm{Ti}) \mathrm{O}_{3} / \mathrm{Metglas}$ laminates under zero magnetic bias is reported. The remanent magnetization was increased by annealing Metglas, which generated an internal bias field. This shifted the $M-H$ hysteresis loops, yielding large values for the ME voltage coefficient of $\alpha_{\mathrm{ME}}=12 \mathrm{~V} / \mathrm{cm} \cdot \mathrm{Oe}$ and $380 \mathrm{~V} / \mathrm{cm} \cdot \mathrm{Oe}$ at $1 \mathrm{kHz}$ and electromechanical resonance under zero magnetic bias, respectively. This self-biased laminate is shown to have a high sensitivity to ac magnetic fields. (C) 2013 American Institute of Physics. [http://dx.doi.org/10.1063/1.4794056]
\end{abstract}

The magnetoelectric (ME) effect- the induction of magnetization by an applied electric field $(E)$, or polarization by magnetic field $(H)$ - has been of recent research interest due to many potential applications. ${ }^{1-3}$ The ME effect in laminate composites is known to be much higher than in single phase and particulate composites, due to a combination of the magnetostrictive and piezoelectric effects of the individual layers. ${ }^{4-6}$ Application of magnetic field to the laminates produces an elastic strain in the magnetostrictive phase that is stress coupled to that of the piezoelectric one, resulting in an induced voltage. Significantly higher values of the ME voltage coefficient $\left(\alpha_{M E}\right)$ have been reported in tri-layer Metglas/ piezo-fibers/Metglas structures with a multi-push-pull configuration that is longitudinally poled and longitudinally magnetized (L-L), promising applications such as magnetic field sensors and gradiometers.

The strength of the ME effect is determined by the piezoelectric $\left(d_{\mathrm{p}}\right)$ and piezomagnetic $\left(d_{m}\right)$ coefficients. To achieve the highest value of $\alpha_{\mathrm{ME}}$, all previously reported ME laminated composites operated in a passive mode have required a magnetic bias field to get the value of $d_{\mathrm{m}}$ to be maximum. In sensor applications, the need of an external magnetic bias presents some significant disadvantages: such as, increasing the required volume and adding a potential noise source, amongst others. ${ }^{7}$ It has recently been reported that epitaxial thin layer ME composites with an additional exchange bias layer have large ME effects at zero magnetic bias. ${ }^{8}$ Such thin layer ME heterostructures are difficult to fabricate and potentially costly compared to laminated ones. Magnetization-graded ME composites have also shown nonzero values of $\alpha_{\mathrm{ME}}$ under zero magnetic bias, but the values of $\alpha_{\mathrm{ME}}$ were small. ${ }^{9,10}$

In this letter, we report a shift in the $M-H$ hysteresis loop for annealed Metglas/Metglas bilayers. As a consequence, giant $\mathrm{ME}$ effects were obtained under zero magnetic bias in self-biased annealed Metglas/ $\mathrm{Pb}(\mathrm{Zr}, \mathrm{Ti}) \mathrm{O}_{3}$ (PZT)/Metglas laminates both at low frequency and at the electromechanical resonance (EMR) condition. Such self-biased ME laminate sensors had a high magnetic field sensitivity.
Metglas foils (Vitrovac $7600 \mathrm{~F}$, Hanau, German) of dimensions $40 \mathrm{~mm} \times 5 \mathrm{~mm}$ were annealed at $600^{\circ} \mathrm{C}$ for $4 \mathrm{~h}$ in air. Figure 1 shows X-ray diffraction (XRD) line scans for amorphous and annealed Metglas. No obvious peaks were observed for the amorphous Metglas. However, after annealing, there were intense peaks for $\alpha$-Fe and hcp-Co near $2 \theta=44^{\circ}$, indicating that the amorphous Metglas had partially crystallized. In addition, less intense peaks were apparent for the iron oxide phases $\left(\mathrm{Fe}_{3} \mathrm{O}_{4}\right.$ and $\left.\mathrm{Fe}_{2} \mathrm{O}_{3}\right)$ at $2 \theta=34^{\circ}$ and $51^{\circ}$, indicating that the surface of the Metglas foil had oxidized. These crystalline and oxidized phases have "harder" magnetic properties than amorphous Metglas. ${ }^{11,12}$ After being magnetized by a dc magnetic bias, these crystalline and oxidized phases can induce a unidirectional magnetic anisotropy in adjacent amorphous Metglas layers. ${ }^{13,14}$

Two layers of Metglas and two layers of annealed Metglas both of dimensions $40 \mathrm{~mm} \times 5 \mathrm{~mm}$ were then epoxied together. After premagnetizing with a bias of $H_{\mathrm{dc}}=+2 \mathrm{kOe}$, the $M-H$ hysteresis loops were measured by a vibrating sample magnetometer (VSM). A high-field $M-H$ loop is given in Figure 2(a). The data in this figure can be seen to consist of two sections: (i) a narrow low-field magnetization process for the "soft" magnetic phase of the amorphous Metglas, and (ii) a wider one for the "hard" annealed

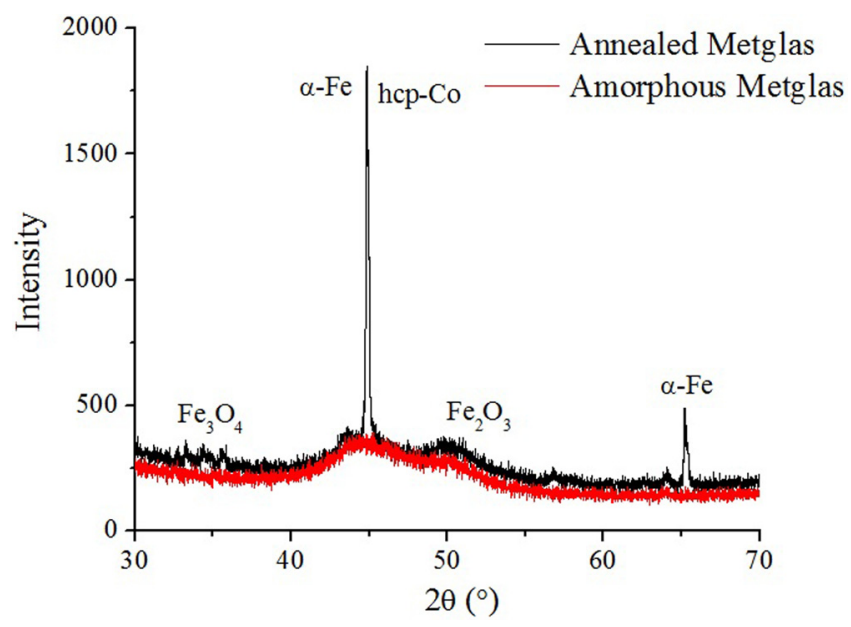

FIG. 1. XRD line scan of amorphous and annealed Metglas foils.

${ }^{\text {a)} 1 i m h 09 @ v t . e d u . ~}$ 

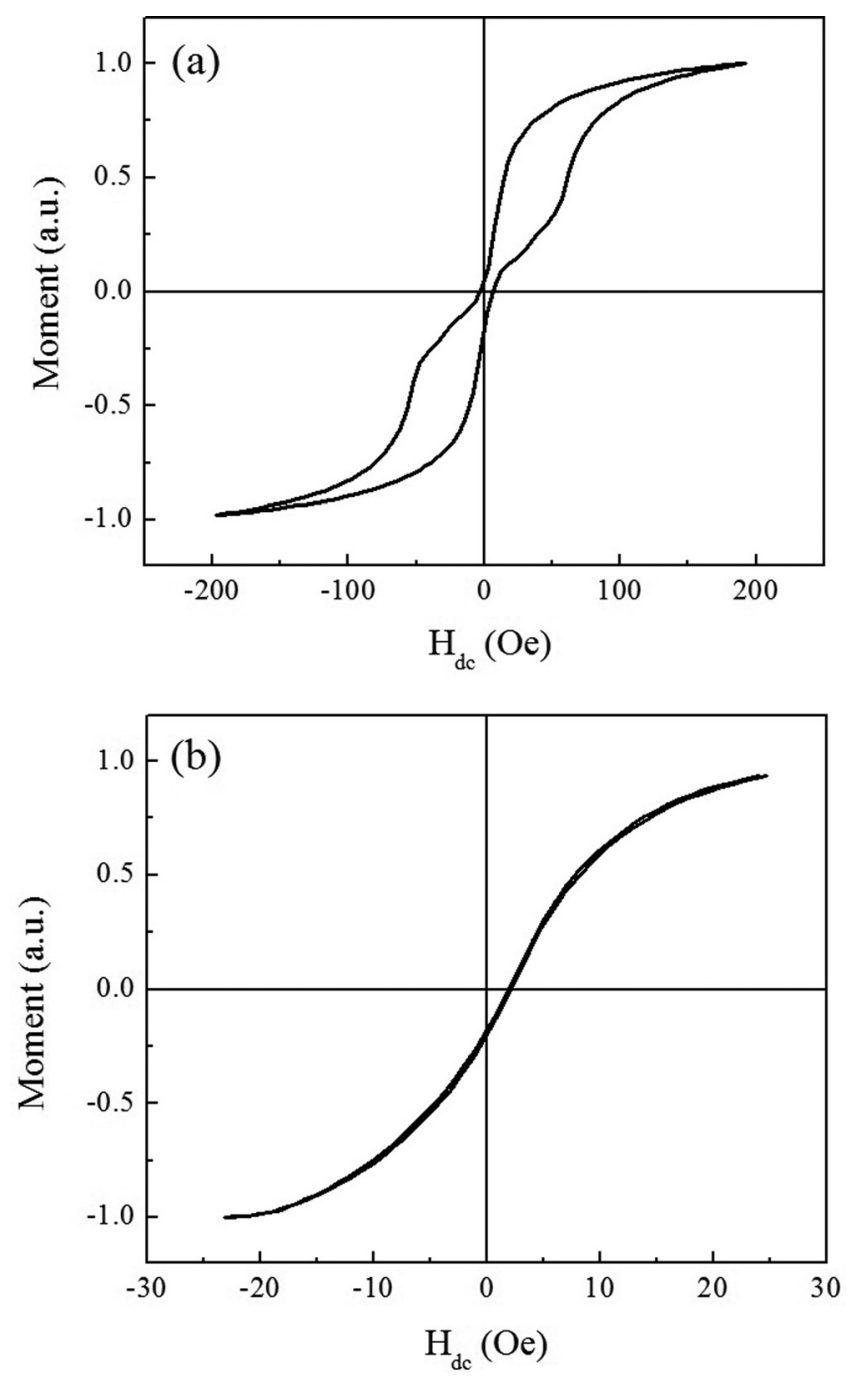

FIG. 2. (a) High-field and (b) low-field $M-H$ hysteresis loops by VSM for two layers of Metglas and two layers of annealed Metglas epoxied together.

Metglas. The low-field $M-H$ loop is given in Figure 2(b), where "soft" magnetic characteristics with a low coercive field can be seen. There was an obvious shift of the $M-H$ loop to positive fields, with a built-in bias of $H_{\mathrm{b}}=2.7$ Oe. Such a shift in the $M-H$ curve has been shown to be mainly due to magnetic interactions between "soft" and "hard" phases. ${ }^{15}$ Accordingly, premagnetizing the annealed Metglas layers creates an internal antiparallel magnetic field in the "soft" amorphous Metglas layers with respect to the "hard" ones, resulting in a $2.7 \mathrm{Oe}$ shift of the $M-H$ loops. These results indicate that an annealed and normal Metglas bilayer structure could be used in ME laminates, yielding a non-zero value of $\alpha_{\mathrm{ME}}$ under $H_{\mathrm{dc}}=0$.

Next, several Metglas/PZT/Metglas multi-push-pull L-L mode laminates were made. A $40 \mathrm{~mm} \times 10 \mathrm{~mm}$ PZT bundle served as the core, which consisted of five $40 \mathrm{~mm} \times 2 \mathrm{~mm}$ PZT-5 A fibers (Smart Materials, Sarasota, FL) oriented along the length direction of the laminates. Two interdigitated (ID) Kapton electrodes were bonded to the top and bottom surfaces of the piezoelectric bundle with epoxy resin (Stycast 1264, USA). ${ }^{16}$ The width and the separation of the electrodes were chosen to be $0.15 \mathrm{~mm}$ and $1 \mathrm{~mm}$, respectively. Two layers of Metglas of dimensions $80 \mathrm{~mm} \times 10 \mathrm{~mm}$

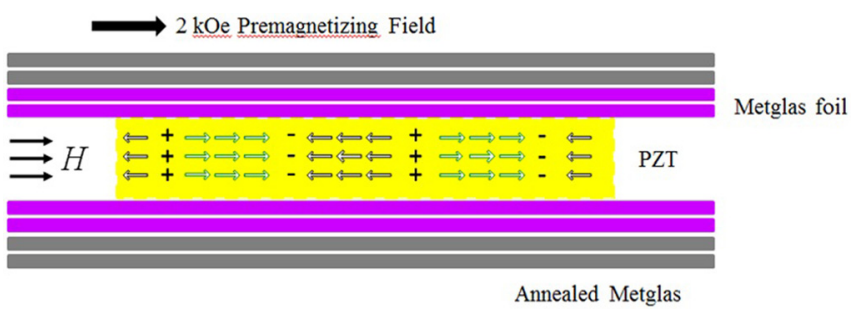

FIG. 3. Schematic of Metglas/PZT/Metglas laminate consisting of annealed Metglas foils. The ID electrodes and epoxy layers are not shown.

were then laminated to both the top and bottom surfaces of the ID electrode/PZT core composites. Two layers of annealed Metglas also of dimensions $80 \mathrm{~mm} \times 10 \mathrm{~mm}$ were then laminated to both surfaces. A schematic of this Metglas/ PZT/Metglas laminate using annealed Metglas foils is shown in Figure 3.

The value of $\alpha_{\mathrm{ME}}$ was then measured using a lock-in amplifier (SR-850) in response to a pair of Helmholtz coils driven at an ac magnetic field of $H_{\mathrm{ac}}=0.1 \mathrm{Oe}$ at a frequency of $f=1 \mathrm{kHz}$. A dc magnetic field was applied by a large electromagnet. Figure 4(a) shows the values of $\alpha_{M E}$ as a
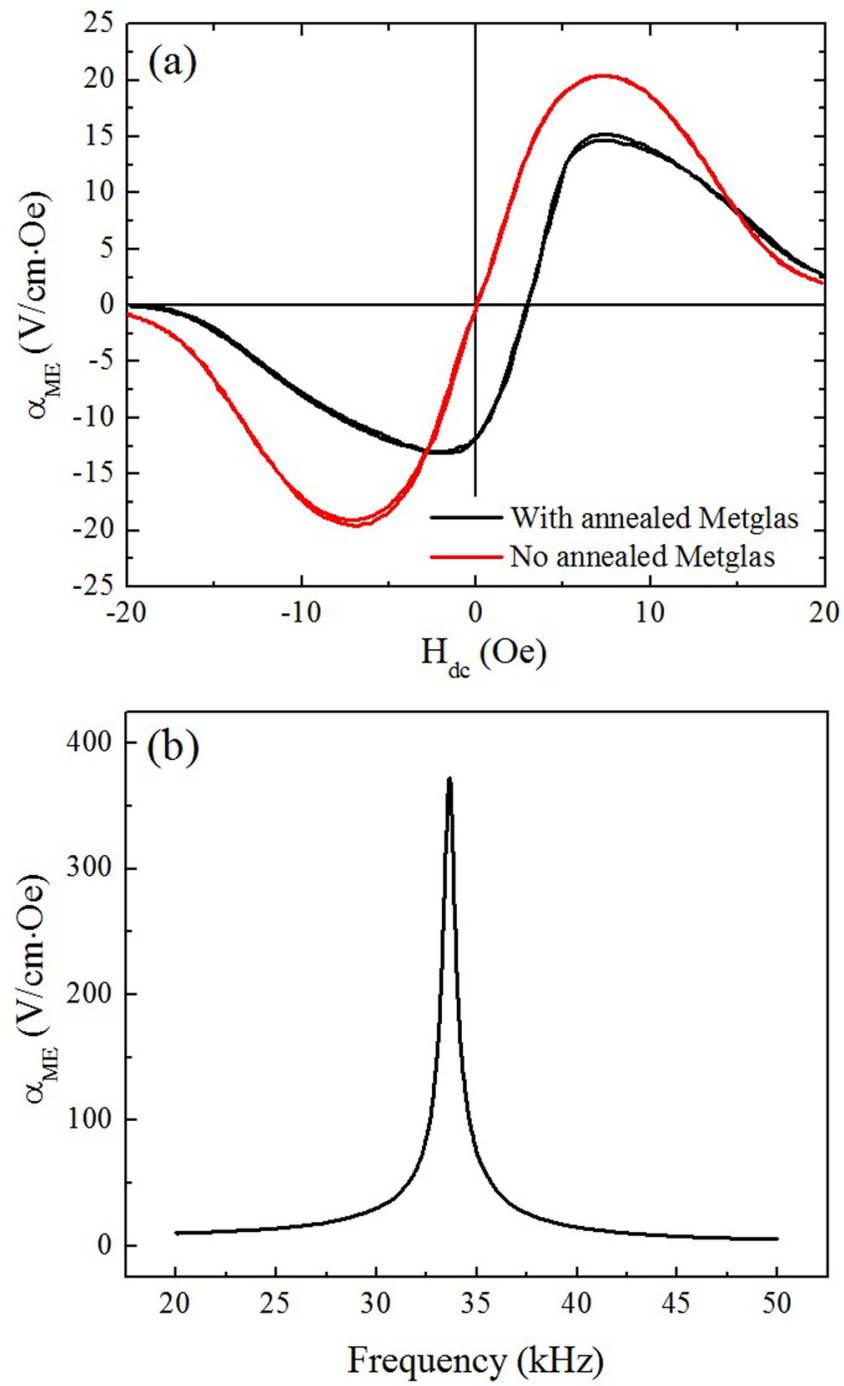

FIG. 4. (a) Value of $\alpha_{\mathrm{ME}}$ as a function of $H_{\mathrm{dc}}$. These data were measured at an ac magnetic field of $H_{\mathrm{ac}}=0.1 \mathrm{Oe}$ and at a frequency of $f=1 \mathrm{kHz}$. (b) Value of $\alpha_{\mathrm{ME}}$ as a function of frequency. These data were measured at an ac magnetic field of $H_{\mathrm{ac}}=0.1 \mathrm{Oe}$ and $H_{\mathrm{dc}}=0$. 


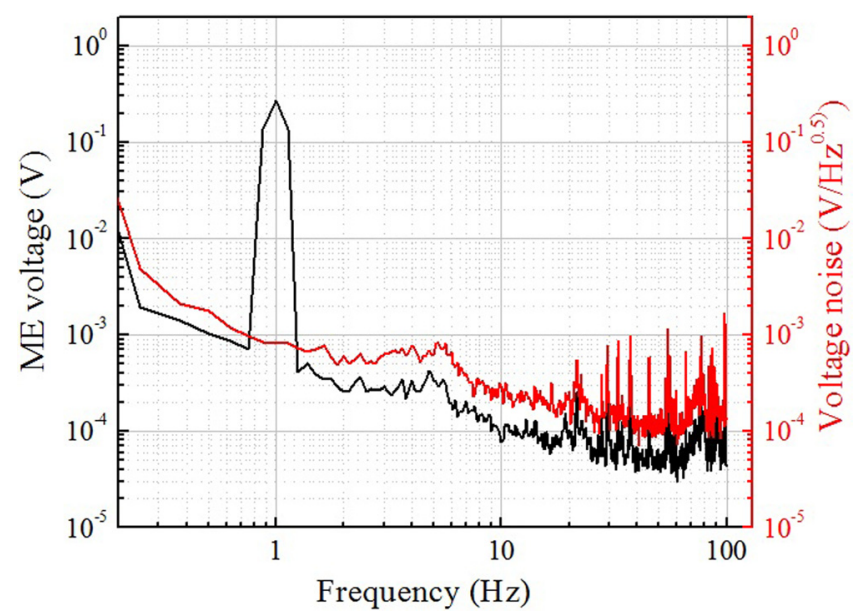

FIG. 5. The output signal of a ME sensor unit containing annealed Metglas foils, and the background voltage noise in the absence of intentional excitation. The incident ac magnetic field was $10 \mathrm{nT}$ at $1 \mathrm{~Hz}$.

function of $H_{\mathrm{dc}}$ for Metglas/PZT/Metglas laminates with and without annealed Metglas foils. For Metglas/PZT/Metglas laminates without annealed Metglas, the values of $\alpha_{\mathrm{ME}}$ were $0 \mathrm{~V} / \mathrm{cm} \cdot \mathrm{Oe}$ at zero magnetic bias and $20 \mathrm{~V} / \mathrm{cm} \cdot \mathrm{Oe}$ under an optimum bias field of 7.5 Oe. For Metglas/PZT/Metglas laminates with annealed Metglas, the curve of $\alpha_{\mathrm{ME}}-H_{\mathrm{dc}}$ response was shifted, resulting in a value of $\alpha_{\mathrm{ME}}=12 \mathrm{~V} / \mathrm{cm} \cdot$ Oe at zero magnetic bias. Please note that the peak value of $\alpha_{\mathrm{ME}}$ was suppressed to $15 \mathrm{~V} / \mathrm{cm}$.Oe, which was a $25 \%$ reduction compared to Metglas/PZT/Metglas laminates without annealed Metglas. The reason for the reduction in the maximum value of $\alpha_{\mathrm{ME}}$ was that the annealed Metglas layers had a lower magnetostriction. A further increase in the layers of annealed Metglas increased the built-in internal bias $H_{\mathrm{b}}$, while reducing the values of $\alpha_{\mathrm{ME}}$ both at peak position and under zero magnetic bias (data not shown). Figure 4(b) shows the values of $\alpha_{\mathrm{ME}}$ as a function of frequency $f$ under $H_{\mathrm{dc}}=0$. The maximum value of $\alpha_{\mathrm{ME}}$ was $380 \mathrm{~V} / \mathrm{cm} \cdot \mathrm{Oe}$ at an EMR frequency of $33.7 \mathrm{kHz}$. Such giant values of $\alpha_{\mathrm{ME}}$ for $H_{\mathrm{dc}}=0$ are much higher than those previously reported for other composites under zero bias, both at low and EMR frequencies.

The laminates were then packaged with a low-noise charge amplifier having a gain of $5.1 \mathrm{~V} \mathrm{pC}^{-1}$ over the frequency range of $0.1<f<100 \mathrm{~Hz}$, which we designate as a ME sensor unit. The unit was placed inside a high-mu-metal magnetic shielded chamber, and connected to a dynamic signal analyzer to measure the voltage noise and output signal. Figure 5 shows a voltage noise spectrum over the frequency range of $0.3<f<100 \mathrm{~Hz}$, which also contained an output signal in response to a $10 \mathrm{nT}$ incident ac magnetic field at $1 \mathrm{~Hz}$. The output of the sensors was $0.27 \mathrm{~V}$, and the background voltage noise at the corresponding frequency was $8.2 \times 10^{-4} \mathrm{~V} / \mathrm{Hz}^{1 / 2}$. Thus, by using the calculation method mentioned in Ref. 17, the magnetic field sensitivity of this self-biased sensor was $30 \mathrm{pT} / \mathrm{Hz}^{1 / 2}$ at $1 \mathrm{~Hz}$ without external bias field. This is in comparison to about $20 \mathrm{pT} / \mathrm{Hz}^{1 / 2}$ at $1 \mathrm{~Hz}$ for ME sensors without annealed Metglas, as previously reported. $^{18}$

In conclusion, giant values of $\alpha_{\mathrm{ME}}$ have been found in self-biased annealed Metglas/PZT/Metglas laminates under zero magnetic field. A magnetic interaction can shift the $M-H$ hysteresis loops in annealed Metglas/Metglas bilayers. In annealed Metglas/PZT/Metglas laminates, values of $\alpha_{\mathrm{ME}}=12 \mathrm{~V} / \mathrm{cm} \cdot \mathrm{Oe}(1 \mathrm{kHz})$ and $380 \mathrm{~V} / \mathrm{cm} \cdot \mathrm{Oe}(\mathrm{EMR})$ were found. This self-biased laminate shows a high sensitivity to small ac magnetic fields.

${ }^{1}$ N. A. Spaldin and M. Fiebig, Science 309, 391 (2005).

${ }^{2}$ R. Ramesh and N. A. Spaldin, Nature Mater. 6, 21 (2007).

${ }^{3}$ C.-W. Nan, M. I. Bichurin, S. Dong, D. Viehland, and G. Srinivasan, J. Appl. Phys. 103, 031101 (2008).

${ }^{4}$ S. Dong, J. Zhai, J. Li, and D. Viehland, Appl. Phys. Lett. 89, 252904 (2006).

${ }^{5}$ J. Zhai, Z. Xing, S. Dong, J. Li, and D. Viehland, J. Am. Ceram. Soc. 91, 351 (2008).

${ }^{6} \mathrm{M}$. Li, Y. Wang, D. Hasanyan, J. Li, and D. Viehland, Appl. Phys. Lett. 100, 132904 (2012).

${ }^{7}$ M. Li, Y. Wang, J. Gao, D. Gray, J. Li, and D. Viehland, J. Appl. Phys. 111, 033923 (2012).

${ }^{8}$ E. Lage, C. Kirchhof, V. Hrkac, L. Kienle, R. Jahns, R. Knochel, E. Quandt, and D. Meyners, Nature Mater. 11, 523 (2012).

${ }^{9}$ S.-C. Yang, C.-S. Park, K.-H. Cho, and S. Priya, J. Appl. Phys. 108, 093706 (2010).

${ }^{10}$ S. K. Mandal, G. Sreenivasulu, V. M. Petrov, and G. Srinivasan, Phys. Rev. B 84, 014432 (2011).

${ }^{11}$ M. Rivas, J. A. Garcóa, M. Tejedor, E. Bertrán, and J. G. Céspedes, J. Appl. Phys. 97, 023903 (2005).

${ }^{12}$ J. He, L. Zhou, D. L. Zhao, and X. L. Wang, J. Mater. Res. 24, 1607 (2009).

${ }^{13}$ L. Zhou, J. He, X. Li, B. Li, D. L. Zhao, and X. L. Wang, J. Phys. D: Appl. Phys. 42, 195001 (2009).

${ }^{14}$ M. Rivas, J. A. García, M. A. Cerdeira, and J. C. Martínez-García, J. NonCryst. Solids 358, 310 (2012).

${ }^{15}$ J. Torrejón, L. Kraus, K. R. Pirota, G. Badini, and M. Vázquez, J. Appl. Phys. 101, 09N105 (2007).

${ }^{16}$ M. Li, D. Berry, J. Das, D. Gray, J. Li, and D. Viehland, J. Am. Ceram. Soc. 94, 3738 (2011).

${ }^{17}$ Y. Wang, D. Gray, D. Berry, J. Gao, M. Li, J. Li, and D. Viehland, Adv. Mater. 23, 4111 (2011).

${ }^{18}$ M. Li, Y. Wang, J. Gao, J. Li, and D. Viehland, Appl. Phys. Lett. 101, 022908 (2012). 